\title{
ACTIVIDAD ANTIMICROBIANA DE CUATRO PLANTAS DEL NOR-ORIENTE PERUANO
}

\author{
Antimicrobial activity of four plants from Peruvian north-east \\ Julio R. Ruiz Q. ${ }^{1}$, Mirtha Roque A. ${ }^{1}$ \\ ${ }^{1}$ Instituto en Química Biológica, Microbiología y Biotecnología "Marco Antonio Garrido Malo" Facultad de Farmacia y \\ Bioquímica, UNMSM.
}

\section{RESUMEN}

Se investigó la actividad antimicrobiana in vitro de los extractos metanólicos, etanólicos e hidroalcohólicos de cuatro plantas del nor-oriente peruano: Cassia reticulata (planta entera), Ilex guayusa Loes (hojas), Piper lineatum (hojas), y Terminalia catappa (hojas). Las especies fueron recolectadas en el departamento de Cajamarca, excepto Terminalia catappa (Amazonas). La actividad antimicrobiana se evaluó mediante el método de difusión en agar. Los microorganismos utilizados fueron las bacterias Staphylococcus aureus, Staphylococcus epidermidis, Pseudomonas aeruginosa, Bacillus subtilis y Escherichia coli; y los hongos Candida albicans, Aspergillus niger y Microsporum canis. De doce extractos investigados, ocho (67\%) presentaron actividad antimicrobiana significativa frente a Staphylococcus aureus y Staphylococcus epidermidis y uno (8\%) frente a Escherichia coli. De doce extractos investigados, diez (83\%) presentaron actividad significativa frente a Candida albicans, y seis (50\%) contra Microsporum canis. Los extractos con la mejor actividad actimicrobiana fueron los tres extractos del Piper lineatum; el extracto hidroalcohólico de Cassia reticulata y el hidroalcohólico de Terminalia catappa.

Palabras clave: Piper lineatum, Cassia reticulata, Terminalia catappa, actividad antimicrobiana, plantas peruanas.

\section{SUMMARY}

The present work investigated the in vitro antimicrobial activities of ethanolic, methonolic and hydroalcoholic extracts corresponding to four plants of north easter of Peru; Cassia reticulata (whole plant), Ilex guayusa Loes (leaves), Piper lineatum (leaves), y Terminalia catappa (leaves). The plants were collected in the department of Cajamarca, except Terminalia catappa (Amazonas). The antimicrobial activity was determinated by the method of agar diffusion. The used microorganisms were the bacteria Staphylococcus aureus, Staphylococcus epidermidis, Pseudomonas aeruginosa, Bacillus subtilis and Escherichia coli; and the fungals Candida albicans, Aspergillus niger and Microsporum canis. Of twelve investigated extracts, eight (67\%) presented significant antimicrobial activity against Staphylococcus aureus and Staphylococcus epidermidis and one (8\%) against Escherichia coli. Of twelve investigated extracts, ten (83\%) presented significant activity against Candida albicans, and six (50\%) against Microsporum canis. The extracts with the greatest antimicrobial activity were the three extracts of Piper lineatum, the hydroalcoholic extract of Cassia reticulata and the hydroalcoholic extract of Terminalia catappa.

Key words: Piper lineatum, Cassia reticulata, Terminalia catappa, antimicrobial activity, peruvian plants.

\section{INTRODUCCIÓN}

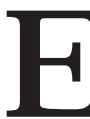
n el Perú, como en otros países en vías de desarrollo, las plantas medicinales representan aún la principal herramienta terapéutica en medicina tradicional. La flora peruana ofrece grandes posibilidades para el descubrimiento de nuevos compuestos con actividad antifúngica. Se calcula que el Perú posee unas 25 ooo especies de plantas conocidas, con 17144 especies de plantas con flores (Angiospermas y Gimnospermas), de las cuales 5354 $(31,3 \%)$ son especies nativas ${ }^{(1)}$.

En los últimos años, después de un período en que la industria farmacéutica se dedicó exclusivamente a la fabricación de fármacos de síntesis, dejando atrás las antiguas medicinas que tenían como base los extractos de plantas medicinales, hay un cambio cualitativo en los programas industriales con dedicación a la búsqueda de nuevos medicamentos de origen herbario. Estos cambios se han sustentado, por una parte, en una filosofía de la vuelta a la naturaleza que impregna el modo de vivir de los países industrializados y, por otra, en necesidades de salud pública, ya que se ha tornado urgente la búsqueda de moléculas para la fabricación de medicamentos antitumorales y anti-SIDA ${ }^{(2)}$.

El avance de la Fitoterapia como disciplina médica ${ }^{(3)}$ es cada vez mayor, esto se evidencia en que las plantas medicinales representan casi el $25 \%$ del total de las prescripciones médicas en países industrializados; en los países en desarrollo la participación de las plantas medicinales en el arsenal terapéutico alcanza el $80 \%{ }^{(4)}$. 
En los últimos años hay un incremento en la incidencia de enfermedades infecciosas, destacando las fúngicas ${ }^{(5)}$ debido al aumento considerable de pacientes inmunocomprometidos, con quimioterapia, con nutrición parenteral, sometidos a cirugía de transplante y el uso de agentes antimicrobianos de amplio espectro, agregados a la presencia de SIDA, lo que da lugar a verdaderas "placas Petri vivientes" individuales, quienes son altamente susceptibles a las infecciones oportunistas. Las infecciones fúngicas sistémicas y dérmicas son causas de alta morbi-mortalidad en este tipo de pacientes, siendo las dermatomicosis problemas serios para niños de las naciones del tercer mundo como consecuencia del deficiente cuidado sanitario ${ }^{(6)}$. Los fármacos disponibles tienen una toxicidad importante, producen recurrencia o causan resistencia, razón por lo cual se está procurando el uso de nuevos agentes antifúngicos más potentes pero sobretodo más seguros que los existentes. Desafortunadamente, las células fúngicas y humanas no son diferentes, comparten gran parte de las vías del metabolismo intermediario, utilizan enzimas muy similares y no es fácil encontrar blancos que ofrezcan la selectividad requerida para obtener un antifúngico seguro ${ }^{(7)}$.

En tal sentido, se escogieron cuatro plantas del nor-oriente peruano, basando su selección en criterios etnomedicinales y quimiotaxonómicos.

El presente trabajo fue realizado en el Instituto de Investigación de Química Biológica, Microbiología y Biotecnología "Marco Antonio Garrido Malo" de la Facultad de Farmacia y Bioquímica de la Universidad Nacional Mayor de San Marcos.

El objetivo del trabajo de investigación fue determinar la actividad antimicrobiana de los extractos etanólicos, metanólicos e hidroalcohólicos de alguna(s) parte(s) o de la planta entera según corresponda, de las especies seleccionadas mediante la prueba de difusión en agar.

\section{MATERIAL Y MÉTODOS}

\section{Recolección de la muestra}

Las plantas Cassia reticulata, Ilex guayusa Loes y Piper lineatum, fueron recolectadas en la Provincia de San Ignacio, Región de Cajamarca, entre los 900 y 1400 msnm, y la Terminalia catappa en el Región de Amazonas, Provincia de Luya a $1200 \mathrm{msnm}$. La recolección e identificación de las especies botánicas fue realizada por el Biólogo Jorge Campos. Los datos etnobotánicos y de recolección se resumen en la Tabla 1.

\section{Preparación de los extractos}

Las partes de las plantas elegidas y secas, se pulverizaron. La extracción se realizó por maceración a temperatura ambiente con etanol al 95\%, metanol y solución hidro-alcohólica. Luego el solvente fue evaporado a sequedad en rotavapor a temperatura menor a $40^{\circ} \mathrm{C}$ (8). Para el bioensayo, los extractos fueron resuspendidos en dimetilsulfóxido (DMSO), a una concentración de $25 \mathrm{mg} / \mathrm{mL}$.

\section{Microorganismos}

Las bacterias y hongos fueron suministradas por el Instituto en Química Biológica, Microbiología y Biotecnología "Marco Antonio Garrido Malo" Facultad de Farmacia y Bioquímica, UNMSM. Se trabajó con las siguientes bacterias: Staphylococcus aureus ATCC 25933, Staphylococcus epidermidis cepa clínica, Bacillus subtilis cepa ambiental, Escherichia coli cepa clínica y Pseudomonas aeruginosa ATCC 27853. Además se trabajaron con los siguientes hongos Candida albicans ATCC 10231, Aspergillus niger ATCC 16404 y Microsporum canis cepa clínica.

\section{Actividad antimicrobiana}

Se realizó mediante la prueba de difusión en agar (8). Esta prueba se basa en la inhibición del crecimiento fúngico, mediante la difusión de sustancias activas en un medio sólido, y posteriormente se evidencia por la formación de halos claros. El medio usado fue agar Müller Hinton (Merck) para las bacterias, agar dextrosa Sabouraud (Merck) para Candida albicans y Aspergillus niger, y agar selectivo para dermatofitos según Taplin (Merck), para Microsporum canis. A $20 \mathrm{~mL}$ del agar específico fundido $\left(45^{\circ} \mathrm{C}\right)$ se le mezcló asépticamente, de un lado con $100 \mu \mathrm{L}$ de una suspensión bacteriana $\left(3 \times 10^{8} \mathrm{UFC} / \mathrm{mL}\right)$ o con $1 \mathrm{~mL}$ de suspensión fúngica $\left(1 \times 10^{4} \mathrm{UFC} / \mathrm{ml}\right)$ y vertidas en placas Petri estériles. Una vez que el agar solidificó se hicieron hoyos con la ayuda de un sacabocado de $11 \mathrm{~mm}$ de diámetro externo, se agregaron $100 \mu \mathrm{L}$ de los diferentes extractos $(25 \mathrm{mg} / \mathrm{mL})$ a los pozos y las placas fueron incubadas por $24 \mathrm{~h} \mathrm{a} 37^{\circ} \mathrm{C}$ para bacterias y $24-72 \mathrm{~h}$ a temperatura ambiente para hongos, usándose como controles positivos oxacilina ( $1 \mu \mathrm{g} / \mathrm{disco})$, norfloxacino (1o $\mu \mathrm{g} / \mathrm{disco})$ y cloranfenicol ( $30 \mu \mathrm{g} /$ disco) para las bacterias, nistatina y ketoconazol para los hongos a una concentración de $0,2 \mathrm{mg} / \mathrm{mL}$ disueltos en DMSO. Los ensayos se llevaron a cabo por triplicado. La actividad antimicrobiana fue medida como el diámetro $(\mathrm{mm})$ del halo de inhibición del crecimiento bacteriano. 


\section{Evaluación fitoquímica preliminar}

Se realizó mediante pruebas fitoquímicas de caracterización ${ }^{(9)}$. Entre los metabolitos secundarios buscados tenemos: quinonas, taninos, compuestos fenólicos, alcaloides, esteroides, y flavonoides.

\section{RESULTADOS}

La Tabla 1 resume los datos etnobotánicos de las plantas seleccionadas. En la Tabla 2, se resume los resultados de la actividad antimicrobiana de los extractos crudos.

Los extractos de plantas que mostraron actividad antimicrobiana significativa (definida como una zona clara de inhibición $\geq 18 \mathrm{~mm}$ ) fueron: extracto hidroalcohólico de Cassia reticulata; extractos etanólico, metanólico e hidroalcohólico de Piper lineatum y extracto hidroalcohólico de Terminalia catappa. Las especies más susceptibles fueron Candida albicans (83\%), Staphylococcus aureus (67\%), Staphylococcus epidermidis (67\%) y Microsporum canis (50\%). Por otro lado solo un extracto fue activo contra Escherichia coli y ninguno contra Pseudomonas aeruginosa, Bacillus subtilis y Aspergillus niger. La zona de inhibición más grande fue desarrollada por el extracto etanólico de las hojas Piper lineatum y por el extracto hidroalcohólico de las hojas de Terminalia catappa, con un halo de $50 \mathrm{~mm}$ y más de $50 \mathrm{~mm}$, respectivamente, contra Microsporum canis.

Los solventes usados (etanol, metanol y DMSO) como disolventes para los extractos crudos no mostraron resultados positivos, demostrando que ellos no influyen en la actividad antimicrobiana mostrada por los extractos de las plantas (Tabla 2).

El estudio fitoquímico preliminar revela una abundante presencia de compuestos fenólicos seguida de alcaloides.

\section{DISCUSIÓN}

Las plantas investigadas son usadas de diversa manera en la medicina popular en la zona de recolección, tal como se observa en las tablas respectivas; muchas de ellas se usan en problemas dérmicos e infecciosos. Los resultados de la prueba de difusión en agar nos demuestran que el $100 \%$ de las plantas investigadas tienen actividad antimicrobiana principalmente contra Candida albicans, Staphylococcus aureus y Staphylococcus epidermidis. Esto avala el potencial que tienen estas plantas como antimicrobianos; así como una acertada selección basada en gran parte en su quimiotaxonomía y uso etnomedicinal.

Los extractos etanólico, metanólico e hidroalcohólico de la planta entera de Cassia reticulata (retama) mostraron actividad significativa contra Staphylococcus aureus, Staphylococcus epidermidis y Candida albicans, excepto el extracto metanólico contra Staphylococcus epidermidis. Los extractos no muestran actividad significativa contra el resto de microorganismos ensayados. Estos resultados son similares a los reportados para Cassia auriculata con respecto a la actividad antibacteriana ${ }^{(10)}$, y contra Candida albicans por Cassia alata y Cassia spectabilis ${ }^{(11,12)}$ y con respecto a la actividad contra dermatofitos se ha reportado actividad del extracto etanólico de la corteza y hojas de Cassia grandis ${ }^{(13)}$ y del extracto acuoso de Cassia grandis y Cassia occidentalis ${ }^{(14)}$.

Los extractos metanólico e hidroalcohólico de las hojas de Ilex guayusa Loes mostrarón actividad significativa contra Candida albicans, y sólo el hidroalcohólico contra Microsporum canis. El resto de extractos de esta planta no muestran actividad significativa contra los otros microorganismos ensayados. Se ha reportado actividad contra Candida albicans por parte de los triterpenos aislados de las frutas de Ilex integras ${ }^{(15)}$. El análisis fitoquímico preliminar reveló la presencia de taninos, compuestos fenólicos, alcaloides, flavonoides, glicósidos y quinonas.

Los extractos etanólico, metanólico e hidroalcohólico de las hojas de Piper lineatum (luto) mostraron actividad significativa contra Staphylococcus aureus, Staphylococcus epidermidis, Candida albicans y Microsporum canis. Los extractos no muestran actividad significativa contra el resto de microorganismos ensayados. Es importante destacar la muy buena actividad contra el Microsporum canis con un halo de inhibición de $50 \mathrm{~mm}$, por parte del extracto hidroalcohólico de las hojas de la Piper lineatum. Para esta especie es la primera vez que se reportan las actividades citadas; sin embargo, este género es conocido por su actividad antimicrobiana. Así tenemos resultados similares en cuanto a la actividad antibacteriana contra Gram positivos del extracto de las partes aéreas del Piper aduncum ${ }^{(16)}$, del extracto en acetato de etilo de las hojas de Piper regnellii ${ }^{(17)}$ y del extracto metanólico de Piper solmsianum ${ }^{(18)}$. Con respecto a la actividad antifúngica, otras especies del género comparten esta actividad, entre ellas tenemos Piper crassinervium Kunth, Piper lanceaefolium HBK, Piper angustifolium, Piper guineense, Piper tuberculatum, Piper arboreum, Piper hispidum, Piper fulvescens, y Piper coruscans $^{(19-28)}$. El análisis fitoquímico preliminar reve- 
Tabla 1. Datos Etnobotánicos de las Plantas Investigadas.

\begin{tabular}{|c|c|c|c|c|c|}
\hline $\begin{array}{l}\text { Nombre } \\
\text { científico }\end{array}$ & Familia & $\begin{array}{l}\text { Nombre } \\
\text { común }\end{array}$ & $\begin{array}{l}\text { Parte } \\
\text { usada }\end{array}$ & $\begin{array}{l}\text { Sitio de } \\
\text { recolección* }\end{array}$ & Usos populares** \\
\hline Cassia reticulata & Fabaceae & Retama & $\begin{array}{l}\text { Planta } \\
\text { entera }\end{array}$ & San Ignacio & $\begin{array}{l}\text { Tratamiento de la fiebre amarilla, sarna y } \\
\text { las manchas blancas de la piel. }\end{array}$ \\
\hline Ilex guayusa Loes & Aquifoliaceae & Guayusa & Hojas & San Ignacio & $\begin{array}{l}\text { Es estimulante del sistema nervioso, para } \\
\text { la infertilidad y para resfrios comunes }\end{array}$ \\
\hline Piper lineatum & Piperaceae & Luto & Hojas & San Ignacio & $\begin{array}{l}\text { Desinflamante, cicatrizante de heridas } \\
\text { externas y procesos de úlceras gástricas; } \\
\text { para lavados vaginales en casos de infla- } \\
\text { maciones por micosis }\end{array}$ \\
\hline Terminalia catappa & Combretaceae & Castañilla & Hojas & Luya & $\begin{array}{l}\text { Como hipotensor, para la diarrea, como } \\
\text { hipocolesterolémico }\end{array}$ \\
\hline
\end{tabular}

* Sitios de resolección: San Ignacio, Región de Cajamarca y Luya, Región de Amazonas

** Basado en los datos obtenidos en los viajes de recolección

Tabla 2. Actividad Antimicrobiana de los Extractos Crudos.

\begin{tabular}{|c|c|c|c|c|c|c|c|c|c|c|}
\hline \multirow{3}{*}{$\begin{array}{l}\text { Especies de } \\
\text { plantas }\end{array}$} & \multirow{3}{*}{$\begin{array}{c}\text { Partes de } \\
\text { plantas } \\
\text { ensayadas }\end{array}$} & \multirow{3}{*}{$\begin{array}{l}\text { Tipo de } \\
\text { extracto }^{b}\end{array}$} & \multicolumn{8}{|c|}{ Diámetro de la zona de inhibición (mm) } \\
\hline & & & \multicolumn{5}{|c|}{ Actividades antibacterianas ${ }^{c}$} & \multicolumn{3}{|c|}{$\begin{array}{c}\text { Actividades } \\
\text { antifúngicas }^{\text {d }}\end{array}$} \\
\hline & & & B.s. & S.a. & S.e. & E.c. & P.a. & C.a. & A.n. & M.c. \\
\hline \multirow[t]{3}{*}{ Cassia reticulata } & PA & $\mathrm{E}$ & 14 & 20 & 19 & 0 & 13 & 21 & 0 & 15 \\
\hline & PA & M & 16 & 18 & 16 & 14 & 12 & 20 & 0 & 0 \\
\hline & PA & HA & 16 & 20 & 21 & 19 & 14 & 23 & 0 & 0 \\
\hline \multirow[t]{3}{*}{ Ilex guayusa Loes } & $\mathrm{H}$ & $\mathrm{E}$ & 0 & 12 & 12 & 13 & 12 & 16 & 0 & 0 \\
\hline & $\mathrm{H}$ & M & 0 & 16 & 12 & 13 & 12 & 24 & 0 & 14 \\
\hline & $\mathrm{H}$ & HA & 14 & 15 & 18 & 15 & 11 & 25 & 0 & 32 \\
\hline \multirow[t]{3}{*}{ Piper lineatum } & $\mathrm{H}$ & $\mathrm{E}$ & 17 & 20 & 18 & 12 & 11 & 22 & 0 & 50 \\
\hline & $\mathrm{H}$ & M & 16 & 23 & 23 & 11 & 0 & 26 & 0 & 44 \\
\hline & $\mathrm{H}$ & HA & 16 & 21 & 20 & 0 & 16 & 23 & 0 & 44 \\
\hline \multirow[t]{3}{*}{ Terminalia catappa } & $\mathrm{H}$ & $\mathrm{E}$ & 13 & 13 & 21 & 11 & 13 & 18 & 0 & 25 \\
\hline & $\mathrm{H}$ & M & 14 & 17 & 20 & 13 & 12 & 17 & 0 & 17 \\
\hline & $\mathrm{H}$ & HA & 13 & 18 & 20 & 12 & 12 & 21 & 0 & 50 \\
\hline \multicolumn{11}{|l|}{ Controles } \\
\hline Etanol 95\% & & & 0 & 0 & 0 & 0 & 0 & 0 & 0 & 0 \\
\hline Metanol & & & 0 & 0 & 0 & 0 & 0 & 0 & 0 & 0 \\
\hline DMSO & & & 0 & 0 & 0 & 0 & 0 & 0 & 0 & 0 \\
\hline Oxacilina (1 ug/disco) & & & 22 & 20 & 13 & & & & & \\
\hline Cloranfenicol (30 ug/disco) & & & & & & 15 & & & & \\
\hline Norfloxacino (10 ug/disco) & & & & & & & 35 & & & \\
\hline Nistatina $(0.2 \mathrm{mg} / \mathrm{ml})$ & & & & & & & & 37 & 40 & \\
\hline Ketoconazol $(0,2 \mathrm{mg} / \mathrm{ml})$ & & & & & & & & 26 & 0 & \\
\hline
\end{tabular}

(a) Partes de plantas ensayadas: PA, partes aéreas; $H$, hojas.

(b) Tipo de extracto : E, etanólico; M, metanólico; HA, hidroalcohólico

(c) Especies de bacterias: B.s., Bacillus subtilis ; S.a., Staphylococcus aureus ; S.e., Staphylococcus epidermidis ; E.c., Escherichia coli ; P.a., Pseudomonas aeruginosa.

(d) Hongos: C.a., Candida albicans ; A.n., Aspergillus niger ; M.c., Microsporum canis. 
Tabla 3. Estudio fitoquímico preliminar de las plantas investigadas.

\begin{tabular}{clcccccccc}
\hline \multirow{2}{*}{$\begin{array}{c}\text { Tipo de } \\
\text { extracto }\end{array}$} & \multicolumn{1}{c}{ Especie vegetal } & Flavonoides & Glicósidos & Alcaloides & Fenólicos & Quinonas & Taninos \\
\cline { 2 - 9 } & Shinoda & $\begin{array}{c}\mathbf{H}_{2} \mathbf{S O}_{4} \\
\boldsymbol{\alpha}_{\text {-naftol }}\end{array}$ & Dragendorff & Mayer & Cl. Férrico & NaOH 5\% & Gelatina \\
\hline \multirow{2}{*}{ EM } & Cassia reticulata Wild (PE) & $(+)$ & $(+)$ & $(+)$ & $(++)$ & $(++)$ & $(+)$ & $(+)$ \\
& $\begin{array}{l}\text { Piper lineatum }(\mathrm{H}) \\
\end{array}$ & $(+)$ & $(+)$ & $(+)$ & $(+)$ & $(+++)$ & $(-)$ & $(+)$ \\
& Terminalia catappa $(\mathrm{H})$ & $(+)$ & $(+)$ & $(++)$ & $(++)$ & $(+++)$ & $(+)$ & $(+)$ \\
& Cassia reticulata Wild (PE) & $(+)$ & $(+)$ & $(++)$ & $(++)$ & $(+++)$ & $(++)$ & $(++)$ \\
EE & Piper lineatum $(\mathrm{H})$ & $(+)$ & $(+)$ & $(+++)$ & $(+++)$ & $(+++)$ & $(+)$ & $(+)$ \\
& Terminalia catappa $(\mathrm{H})$ & $(-)$ & $(+)$ & $(++)$ & $(+)$ & $(+++)$ & $(+)$ & $(-)$ \\
& Cassia reticulata Wild $(\mathrm{PE})$ & $(++)$ & $(+)$ & $(+)$ & $(+)$ & $(+++)$ & $(+)$ & $(+)$ \\
\multirow{2}{*}{ EHA } & Ilex guayusa Loes & $(+)$ & $(-)$ & $(+)$ & $(+)$ & $(+++)$ & $(-)$ & $(+)$ \\
& Piper lineatum $(\mathrm{H})$ & $(++)$ & $(++)$ & $(++)$ & $(++)$ & $(+++)$ & $(-)$ & $(+)$ \\
& Terminalia catappa $(\mathrm{H})$ & $(++)$ & $*$ & $(+)$ & $(++)$ & $(+++)$ & $(-)$ & $(-)$ \\
\hline
\end{tabular}

Partes estudiadas: $\mathrm{H}$, hojas; PE, planta entera

* No realizado

Tipo de extracto: EM, extracto etanólico; EE, extracto etanólico; EHA, extracto hidroalcohólico

la la presencia de taninos y compuestos fenólicos entre sus principales constituyentes, los cuales pueden ser los responsables de la actividad biológica. En este género se han reportado los siguientes compuestos antifúngicos: hidroquinonas preniladas y sakuretina, con actividades comparables a los controles (nistatina y miconazol) ${ }^{(20)}$, canfor y canfeno principales constituyentes del aceite esencial de Piper angustifolium ${ }^{(21)}$, numerosas amidas (23-25), derivados del ácido benzoico ${ }^{(26)}$, neolignanos ${ }^{(27,}$ ${ }^{29)}$, y derivados ciclopentanodionas (coruscanona A y B) (28). Todos estos resultados remarcan el gran potencial del género en este campo de la investigación, el cual debe ser profundizado.

Los extractos etanólico, metanólico e hidroalcohólico de las hojas de Terminalia catappa (castañilla) mostraronactividad significativa contra Staphylococcus epidermidis, Candida albicans y Microsporum canis, excepto por los extractos metanólicos contra Candida albicans y Microsporum canis.

El resto de extractos no muestran actividad significativa contra los otros microorganismos ensayados. Es importante destacar la muy buena actividad contra el Microsporum canis con un halo de inhibición de 50 $\mathrm{mm}$, por parte del extracto hidroalcohólico de las hojas de la Terminalia catappa. Los hallazgos concuerdan con lo reportado en la literatura; así tenemos que se ha reportado que el extracto etanólico de sus hojas tiene actividad contra bacterias Gram positivas y Gram negativas ${ }^{(16,30)}$, al igual que los extractos de la corteza del tallo y la raíz de Terminalia brownii ${ }^{(31)}$. La literatura indica que, el extracto metanólico y acuoso de las partes aéreas de Terminalia australis mostró efecto contra cepas de Aspergillus y Candida ${ }^{(32)}$. El extracto metanólico de la raíz de Terminalia sambesiaca y el extracto metanólico de la raíz de Terminalia sericea mostrarón actividad anticandida ${ }^{(33)}$. La planta Terminalia avicennioides es rica en taninos y saponinas, lo que explicaría su actividad contra cepas de Candida y dermatofitos (34). De los extractos de frutos de Terminalia bellirica se aislaron lignanos que poseen actividad antifúngica (35). Terminalia sericea y T. brachystemma originarios de Sudáfrica tienen compuestos antifúngicos de naturaleza apolar contra Microsporum canis ${ }^{(36)}$.

\section{CONCLUSIONES}

El estudio de la actividad antimicrobiana de las plantas peruanas, seleccionadas en base a su quimiotaxonomía y su uso etnomedicinal, ha proveído varios extractos con fuerte actividad contra microorganismos patógenos. Los resultados de este estudio soportan el uso folklórico de estas especies. De doce extractos investigados, ocho (67\%) presentaron actividad antibacteriana significativa frente a Staphylococcus aureus y Staphylococcus epidermidis y uno (8\%) frente a Escherichia coli. De doce extractos investigados, diez $(83 \%)$ presentaron actividad significativa frente a Candida albicans, y seis (50\%) contra Microsporum canis. Los extractos con la mejor actividad actimicrobiana fueron los tres extractos del Piper lineatum; el extracto hidroalcohólico de Cassia reticulata y el hi- 
droalcohólico de Terminalia catappa. Se desconoce los compuestos responsables de su actividad biológica, para lo cual son necesarios estudios posteriores.

\section{REFERENCIAS BIBLIOGRÁFICAS}

1. Brack A. Diccionario Enciclopédico de plantas útiles del Perú. Centro Bartolomé de las Casas. Cuzco 1999.

2. Estrella E. Plantas Medicinales Amazónicas: Realidad y Perspectivas Tratado de Cooperación Amazónica: Secretaría Pro Tempore. Lima 1995.

3. Obregón L. Fitoterapia: Importancia de su desarrollo al servicio de salud FITO 2003 Lima.

4. Machado L. Materias primas vegetales para la industria de fitofarmacos FITO 2003 Lima.

5. Navarro-Garcia VM, Gonzalez A, Fuentes M, Aviles M, Rios MY, Zepeda G, Rojas MG. Antifungal activities of nine traditional Mexican medicinal plants. J Ethnopharmacol 2003; 87(1):85-8.

6. Lopez SN, Castelli MV, Zacchino SA, Dominguez JN, Lobo G, Charris-Charris J, et al. In vitro antifungal evaluation and structure-activity relationships of a new series of chalcone derivatives and synthetic analogues, with inhibitory properties against polymers of the fungal cell wall. Bioorg Med Chem 2001; 9(8): 1999-2013.

7. Zacchino S. Estratégias para a descoberta de novos agentes antifúngicos. SC, Brasil: Grifos (Ed), 2001.

8. Rojas R, Bustamante B, Bauer J, Fernández I, Albán J, Lock O. Antimicrobial activity of selected Peruvian medicinal plants J Ethnopharmacol 2003; 88 (2-3): 199-204

9. Ruiz MH. Estudio de la Actividad Antibacteriana y Antiviral de Hypericum laricifolium del Perú [Tesis]. Facultad de Farmacia y Bioquímica UNMSM. Lima; 2003.

10. Duraipandiyan V, Ayyanar M, Ignacimuthu S. Antimicrobial activity of some ethnomedicinal plants used by Paliyar tribe from Tamil Nadu, India. BMC Complement Altern Med 2006 Oct 17; 6: 35

11. Somchit MN, Reezal I, Elysha Nur I, Mutalib AR In vitro antimicrobial activity of ethanol and water extracts of Cassia alata. J Ethnopharmacol 2003; 84(1): 1-4.

12. Sangetha S, Zuraini Z, Sasidharan S, Suryani S. Fungicidal effect and oral acute toxicity of Cassia spectabilis leaf extract. Nippon Ishinkin Gakkai Zasshi 2008; 49(4): 299304.

13. Caceres A. et al. Plants used in Guatemala for the treatment of dermatophytic infections. Evaluation of antifungal activity of seven American plants. J Ethnopharmacol 1993; 40(3): 207-13.

14. Caceres A, Lopez BR, Giron MA, Logemann H. Plants used in Guatemala for the treatment of dermatophytic infections. Screening for antimycotic activity of 44 plant extracts. J Ethnopharmacol 1991; 31(3): 263-76.

15. Haraguchi H, Kataoka S, Okamoto S, Hanafi M, Shibata
K. Antimicrobial triterpenes from Ilex integra and the mechanism of antifungal action. Phytother Res 1999; 13(2): 151-6

16. Kloucek P, Polesny Z, Svobodova B, Vlkova E, Kokoska L. Antibacterial screening of some Peruvian medicinal plants used in Callería District. J Ethnopharmacol 2005 Jun 3; 99(2): 309-12.

17. Pessini GL, Dias Filho BP, Nakamura CV, Cortez DA. Antibacterial activity of extracts and neolignans from Piper regnellii (Miq.) C. DC. var. pallescens (C. DC.) Yunck. Mem Inst Oswaldo Cruz. 2003 Dec; 98(8): 111520.

18. Campos MP, Cechinel Filho V, Silva RZ, Yunes RA, Monache FD, Cruz AB. Antibacterial activity of extract, fractions and four compounds extracted from Piper solmsianum C. DC. VAR. solmsianum (Piperaceae). Z Naturforsch [C]. 2007 Mar-Apr; 62(3-4): 173-8.

19. Lopez A, Hudson JB, Towers GH. Antiviral and antimicrobial activities of Colombian medicinal plants. J Ethnopharmacol 2001 Oct; 77(2-3): 189-96.

2o. Danelutte AP, Lago JH, Young MC, Kato MJ Antifungal flavonones and prenylated hydroquinones from Piper crassinervum Kunth. Phytochemistry 2003; 64: 555-559

21. Tirillini B, Velasquez ER, Pellegrino R. Chemical composition and antimicrobial activity of essential oil of Piper angustifolium. Planta Med 1996; 62(4): 372-3

22. Ngono Ngane A, Biyiti L, Bouchet P, Nkengfack A, Amvam Zollo PH. Antifungal activity of Piper guineense of Cameroon. Fitoterapia 2003 Jul; 74(5): 464-468.

23. Vasques da Silva R, Navickiene HM, Kato MJ, Bolzani Vda S, Meda CI, Young MC, Furlan M. Antifungal amides from Piper arboreum and Piper tuberculatum. Phytochemistry 2002; 59(5): 521-7.

24. Navickiene HM, Alecio AC, Kato MJ, Bolzani VD, Young MC, Cavalheiro AJ, et al. Antifungal amides from Piper hispidum and Piper tuberculatum. Phytochemistry 2000; 55(6): 621-6.

25. Alecio AC, da Silva Bolzani V, Young MC, Kato MJ, Furlan M. Antifungal amide from leaves of Piper hispidum. J Nat Prod 1998; 61(5): 637-9

26. Lopez A, Ming DS, Towers GH. Antifungal activity of benzoic acid derivatives from Piper lanceaefolium. J Nat Prod 2002; 65(1):62-4.

27. Freixa B, Vila R, Ferro EA, Adzet T, Canigueral S. Antifungal principles from Piper fulvescens. Planta Med 2001 Dec; 67(9): 873-5.

28. Li XC, Ferreira D, Jacob MR, Zhang Q, Khan SI, ElSohly $\mathrm{HN}$, et al. Antifungal cyclopentenodiones from Piper coruscans J Am Chem Soc 2004, 126(22): 6872-6873.

29. Johann S, Cota BB, Souza-Fagundes EM, Pizzolatti MG, Resende MA, Zani CL. Antifungal activities of compounds isolated from Piper abutiloides Kunth. Mycoses 2008 Dec 3.

30. Pawar SP, Pal SC. Antimicrobial activity of extracts of Terminalia catappa root. Indian J Med Sci 2002 Jun; 
56(6): 276-8.

31. Mbwambo ZH, Moshi MJ, Masimba PJ, Kapingu MC, Nondo RS. Antimicrobial activity and brine shrimp toxicity of extracts of Terminalia brownii roots and stem. BMC Complement Altern Med 2007 Mar 30; 7: 9.

32. Carpano SM, et al . Antifungal activity of Terminalia australis. Fitoterapia 2003 Apr; 74(3): 294-7.

33. Fyhrquist P, Mwasumbi L, Haeggström CA, Vuorela H, Hiltunen R, Vuorela P. Ethnobotanical and antimicrobial investigation on some species of Terminalia and Combretum (Combretaceae) growing in Tanzania. J Ethnopharmacol 2002 Feb; 79(2): 169-77.

34. Baba-Moussa F, Akpagana K, Bouchet P. Antifungal activities of seven West African Combretaceae used in traditional medicine. J Ethnopharmacol. 1999; 66(3): 335-8.

35. Valsaraj R, et al. New anti-HIV-1, antimalarial, and antifungal compounds from Terminalia bellerica. J Nat Prod 1997 Jul; 6o(7): 739-42.
36. Masoko P, Picard J, Eloff JN. Antifungal activities of six South African Terminalia species (Combretaceae). J Ethnopharmacol 2005 Jun 3; 99(2): 301-8.

Manuscrito recibido el: 30/11/2009

Aceptado para su publicación el:07/o1/2010

\section{CORRESPONDENCIA}

Q.F. Julio Reynaldo Ruiz Quiroz

Profesor Auxiliar del Departamento de Microbiología y Parasitología Básica y Aplicada. Facultad de Farmacia y Bioquímica - UNMSM

Jr. Puno 1002 -Lima 1 (Perú)

E-mail: julioruizquiroz@gmail.com 